\title{
Re-sensitization of radiation resistant colorectal cancer cells to radiation through inhibition of AMPK pathway
}

\author{
HONGYONG JIN $^{1 *}$, SUJIE GAO ${ }^{2 *}$, HUILING GUO $^{1}$, SHENGNAN REN $^{1}$, \\ FUJIAN JI ${ }^{1}$, ZHUO LIU $^{1}$ and XUEBO CHEN ${ }^{1}$ \\ Departments of ${ }^{1}$ General Surgery and ${ }^{2}$ Anesthesia, China Japan Union Hospital of Jilin University, \\ Changchun, Jilin 130033, P.R. China
}

Received January 3, 2015; Accepted February 1, 2016

DOI: $10.3892 / 01.2016 .4339$

\begin{abstract}
Radiotherapy (RT) is commonly used to treat multi-tumors to attenuate the risk of recurrence. Despite impressive initial clinical responses, a large proportion of patients experience resistance to RT. Therefore, identification of functionally relevant biomarkers would be beneficial for radioresistant patients. Adenosine monophosphate-activated kinase (AMPK) is recognized as a mediator of tumor suppressor gene function. In the present study, radio-sensitive and -resistant colon cancer patient samples were compared and the AMPK pathway was observed to be highly activated in radioresistant patients. In addition, the protein and mRNA levels of AMPK were upregulated in radioresistant colon cancer cells in comparison to radiosensitive colon cancer cells. The present study provides evidence that activation of AMPK by metformin contributes to radioresistance. Inhibition of AMPK by either small interfering RNA or Compound C, which is a specific inhibitor of AMPK, re-sensitized radiation resistant cells. The data presented indicates a synergistic effect on radiation resistant cancer cells by the combination of Compound $\mathrm{C}$ and radiation. In summary, the present study proposes that inhibition of the AMPK pathway is a potential strategy for reversing radiation resistance and may contribute to the development of therapeutic anticancer drugs.
\end{abstract}

\section{Introduction}

Radiotherapy (RT) is commonly used to treat multi-cancers, including colorectal, lung, prostate, and breast cancer (1). Radiation may be used prior to or following surgical resection of cancerous tumors to attenuate the risk of recurrence and it is often combined with chemotherapy. While a proportion of

Correspondence to: Dr Xuebo Chen, Department of General Surgery, China Japan Union Hospital of Jilin University, 126 Xiantai Street, Changchun, Jilin 130033, P.R. China

E-mail: chenxuebo1981@126.com

${ }^{*}$ Contributed equally

Key words: radioresistance, AMPK, compound C, colorectal cancer, metformin tumors do respond well initially, a large proportion of patients experience resistance to RT (2). Therefore, it is important to elucidate the molecular basis that contributes to RT resistance in order to screen patients prior to them receiving therapy. In addition, the development of adjuvant treatments to enhance the efficacy of radiation are imperative.

Radiation treatment generates free radicals and reactive oxygen species (ROS) that attack the covalent bonds of DNA, leading to breaks in double stranded DNA (DSBs) (3). In response to DNA damage, the kinase Ataxia Telangiectasia Mutated (ATM) is activated through auto-phosphorylation to stimulate DNA repair pathways (4). ATM also regulates the pro-survival and radio-resistance pathway of Akt-mammalian target of rapamycin (mTOR), through which the cell survival pathway is activated (5).

The energy sensor adenosine monophosphate-activated kinase (AMPK) is a heterotrimeric enzyme composed of one catalytic subunit and two regulatory subunits with critical roles in regulating growth and reprogramming metabolism (6). It is a downstream effector of liver kinase-B 1 (LKB1), a tumor suppressor gene that is mutated in Peutz-Jeghers syndrome (7). AMPK is also a highly conserved sensor of intracellular adenosine nucleotide levels; under energy stress, AMPK is activated through the phosphorylation on Thr172 by LKB1 (8) to restrict energy consuming anabolic processes such as protein synthesis, cell cycle and proliferation instead of the stimulation of substrate uptake and energy generation through processes such as glucose and amino acid uptake. Moreover, AMPK induces cell cycle arrest in response to metabolic stress through induction of p53 (9). It has been reported that AMPK inhibits mTORC1 through phosphorylation of tuberous sclerosis 2 (TSC2) on Ser1387 and phosphorylation of Raptor, an essential component of mTORC1 (10). A recent study demonstrated that AMPK may be activated by irradiation in an LKB1-independent manner through the ATM-AMPK-p53/p21 cip1 signaling pathway that facilitates the irradiation-induced cell cycle arrest at G2-M phase (11), indicating that targeting the AMPK pathway may be a novel strategy for radio-sensitization in human cancer. In the present study, AMPK expression levels were compared in radiation resistant and radiation sensitive colorectal cancer patients. By establishing the radiation resistant cell line from colon cancer cells, the AMPK expression levels were determined in 
radioresistant cells and the role of AMPK inhibitor in reversing the radioresistance was also investigated.

\section{Materials and methods}

Patients and sample. A total of 5 fresh tissue samples, from radiation sensitive and resistant colon cancers, were procured from surgical resection specimens collected by the Division of General Surgery, China Japan Union Hospital of Jilin University (Changchun, China) from March 2012 to May 2013. Primary tumor regions and corresponding histologically normal tissues from the same patients were separated by experienced pathologists, and immediately stored in liquid nitrogen $\left(-193^{\circ} \mathrm{C}\right)$ until use. The use of patient samples was approved by the institutional ethics committee of Radiation Medicine Institute.

Cells culture and ionizing radiation. The human colon cancer cell line DLD-1 was purchased from the American Type Culture Collection (Manassas, VA, USA). Cells were cultured in RPMI-1640 medium (Thermo Fisher Scientific, Inc., Waltham, MA, USA) with added $10 \%$ fetal bovine serum (Thermo Fisher Scientific, Inc.), 2 mM glutamine (Thermo Fisher Scientific, Inc.), $50 \mathrm{IU} / \mathrm{ml}$ penicillin (Thermo Fisher Scientific, Inc.) and $50 \mu \mathrm{g} / \mathrm{ml}$ streptomycin (Thermo Fisher Scientific, Inc.), and were maintained in humidified $37^{\circ} \mathrm{C}, 5 \% \mathrm{CO}_{2}$ incubators. Prior to collection, cultures were tested for mycoplasma infection using Myco Alert (Lonza, Walkersville, MD, USA) according to the manufacturer's protocol. Cells were exposed to different doses of irradiation (IR) using a 60 Co clinical radiation unit (Co-60 T780C; Best Theratronics, Ltd., Kanata, Canada). Cells were pre-incubated with drugs (Metformin, 0.5, 1 and $2 \mu \mathrm{M}$; Compound $\mathrm{C}, 1,2,4$ and $8 \mu \mathrm{M}$ ) for $1 \mathrm{~h}$ before IR, followed by incubation for $1 \mathrm{~h}$ at $37^{\circ} \mathrm{C} 5 \% \mathrm{CO}_{2}$ incubators, then the cells were subjected to downstream analysis. Cells were repeatedly exposed to gradually increasing doses of IR from 1 to $20 \mathrm{~Gy}$ for 4 months, and the surviving cells were collected. Radioresistant DLD-1 cell clones were pooled for the following experiments.

Antibodies and reagents. Antibodies used for this study were: mouse monoclonal AMPK $\alpha 1$ Antibody (H-4) (Santa Cruz Biotechnology, Inc., Dallas, TX, USA; sc-398861); rabbit polyclonal p-AMPKa1/2 Antibody (Thr 172) (Santa Cruz Biotechnology, Inc.; sc-33524); mouse monoclonal GAPDH (Santa Cruz Biotechnology, Inc.; sc-365062). Metformin and Compound $\mathrm{C}$ were purchased from Sigma-Aldrich (Shanghai, China). The secondary antibodies used were as follows: Goat anti-rabbit immunoglobulin (Ig)G, horseradish peroxidase (HRP)-linked antibody (Cell Signaling Technology, Inc., Danvers, MA, USA; 7074) and horse anti-mouse IgG HRP-linked antibody (Cell Signaling Technology, Inc.; 7076).

Reverse transcription-quantitative polymerase chain reaction $(R T-q P C R)$. RNA was isolated from cultured cells using the RNeasy mini-kit (Qiagen GmbH, Hilden, Germany) (with an on-column DNAse digestion step according to the manufacturer's instructions). Briefly, lysates of cells were passed through a Qiashredder (Qiagen $\mathrm{GmbH}$ ) and the eluted lysates were mixed $1: 1$ with $70 \%$ ethanol. The lysates were applied to a mini-column and after washing and DNAse I digestion, the RNA was eluted in 30-50 $\mu \mathrm{l}$ of RNAse-free water. The quantity and quality of total
RNA samples was checked by agarose-gel-electrophoresis and the Bioanalyzer RNA 6000 Nano assay (Agilent Technologies $\mathrm{GmbH}$, Waldbronn, Germany). The sequences of the primers used for RT-qPCR were as follows: AMPK $\alpha 1, \mathrm{~F}$ 5'-CTCAGT TCCTGGAGAAAGATGG-3' and R 5'-CTGCCGGTTGAG TATCTTCAC-3'; and 18S rRNA, F 5'-TGCTGTCCCTGTATG CCTCT-3' and R 5'-TGTAGCCACGCTCGGTCA-3'.

Plasmid DNA and siRNA transfections. Transfection was performed using the Lipofectamine 2000 Transfection reagent (Invitrogen; Thermo Fisher Scientific, Inc.) according to the manufacturer's protocol. Briefly, $12 \mathrm{~h}$ prior to transfection, cells were switched to medium without antibiotics. The transfections were performed when cells reached $80 \%$ confluence, using a 1:3 ratio of DNA $(\mu \mathrm{g})$ to Lipofectamine $(\mu \mathrm{l})$; medium was switched to regular medium $12 \mathrm{~h}$ following transfection. Overexpression vectors containing wild type AMPK $\alpha 1$ were obtained from OriGene Technologies, Inc. (Rockville, MD, USA). siRNA for scramble control and siAMPK $\alpha 1$ were also purchased from Origene Technologies, Inc. The cells were collected or whole-cell lysates were prepared $48 \mathrm{~h}$ after transfection, for further analysis.

Cell viability assay. A total of $5 \times 10^{5}$ cells/well were seeded in 6-well plates and incubated overnight. The medium was replaced, then the cells were treated with either Metformin or Compound $\mathrm{C}$ for $1 \mathrm{~h}$ at the indicated concentrations followed by exposure to radiation (Metformin treatment: $0,1,2,4$, 8 or $16 \mathrm{~Gy}$; Compound $\mathrm{C}$ treatment: $0,0.5,1,2,4$ or $8 \mathrm{~Gy}$ ). The cell viability was determined by trypan blue exclusion test with trypan blue $(0.4 \%)$ purchased from Sigma Chemical Co. (St. Louis, MO, USA).

Western blot analysis. Whole cell extracts were prepared from cultured cells by homogenizing cells in a lysis buffer $(10 \mathrm{mM}$ Tris- $\mathrm{HCl}$ (pH 7.5), $150 \mathrm{mM} \mathrm{NaCl}, 1 \% \mathrm{NP} 40$ ) containing Halt ${ }^{\mathrm{TM}}$ Protease and Phosphatase Inhibitor Cocktail (Thermo Fisher Scientific, Inc.). After centrifugation at 15,000 RCF for $30 \mathrm{~min}$ at $4^{\circ} \mathrm{C}$, supernatants were recovered and used for immunoblot analysis. The proteins were separated by SDS-PAGE and then transferred to polyvinylidene difluoride (PVDF) membranes (Merck Millipore, Darmstadt, Germany). The blots were blocked with 5\% BSA in TBST buffer (Thermo Fisher Scientific, Inc.) and then probed with antibodies against total AMPK $(1: 1,000)$, phosphor-AMPK T172 $(1: 1,000)$ and GAPDH overnight at $4^{\circ} \mathrm{C}$. After washing, the blots were incubated with HRP-conjugated secondary antibodies at room temperature for $1 \mathrm{~h}$ and visualized by super ECL detection reagent (Applygen, Beijing, China).

Statistical analysis. Statistics was evaluated using GraphPad Prism 5.0 software (GraphPad Software, Inc.). The unpaired Student's $t$-test was used for the data analysis, in addition to analysis of variance plus a post-hoc test. All data were shown as mean \pm standard error (SE). $\mathrm{P}<0.05$ was considered to indicate a statistically significant difference.

\section{Results}

AMPK is activated in radiation resistant colon cancer patients. A previous study reported that ionizing radiation induced timeand dose-dependent phosphorylation of AMPK at Thr172 (12). 
A

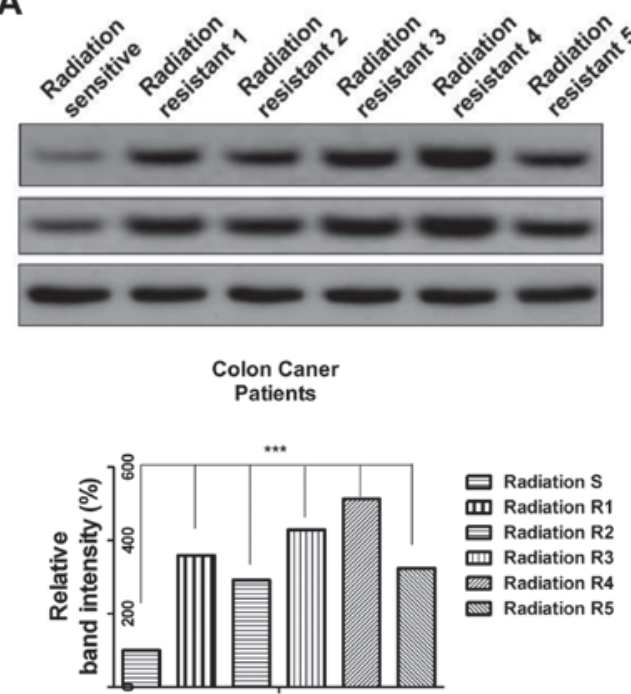

B

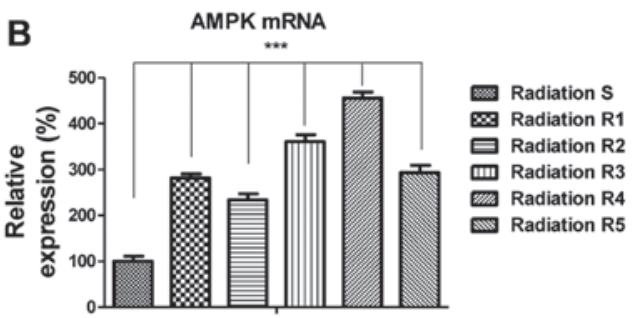

Figure 1. AMPK pathway is upregulated in radiation resistant colon cancer patients. (A) The protein levels of t-AMPK and p-AMPK at T172 in radiation sensitive and resistant colon cancer patients were measured by western blot analysis. GAPDH was used as a loading control. (B) The mRNA levels of AMPK were upregulated in radiation resistant colon cancer patients. Columns, mean of three independent experiments; bars, standard error. ${ }^{* * * *} \mathrm{P}<0.001$. AMPK, adenosine monophosphate-activated kinase; t-AMPK, total AMPK; p-AMPK, phospho-AMPK; GAPDH, glyceraldehyde-3-phosphate dehydrogenase.

To investigate whether the activation of AMPK is clinically correlated with resistance to radiation, the phosphorylation statuses of AMPK and total AMPK were assessed in radioresistant colon cancer patient samples. Notably, phosphorylated AMPK and total AMPK were upregulated in radioresistant patients and radiosensitive patients (Fig. 1A; $\mathrm{P}<0.001$ ), indicating that AMPK may contribute to radiation resistance in colon cancer. In addition, RT-qPCR results demonstrated that the mRNA levels of AMPK were upregulated in radioresistant cancer samples compared with radiosensitive patient samples (Fig. 1B; P<0.001). These results suggest there is a correlation between AMPK and radioresistance, and therefore targeting AMPK may be a potential therapeutic strategy for overcoming tumor radiation resistance.

Radiation resistant colon cancer cells display elevated AMPK activity and expression. To further examine the roles of AMPK in radiation resistance, a radiation resistant colon cancer cell line was generated from DLD-1 parental cells by exposure to repeated irradiation for 2 months. Radioresistant cell clones were selected and pooled for the following experiments. To verify the radioresistance, parental cells (sensitive) and resistant pool cells were irradiated with different doses
A DLD-1

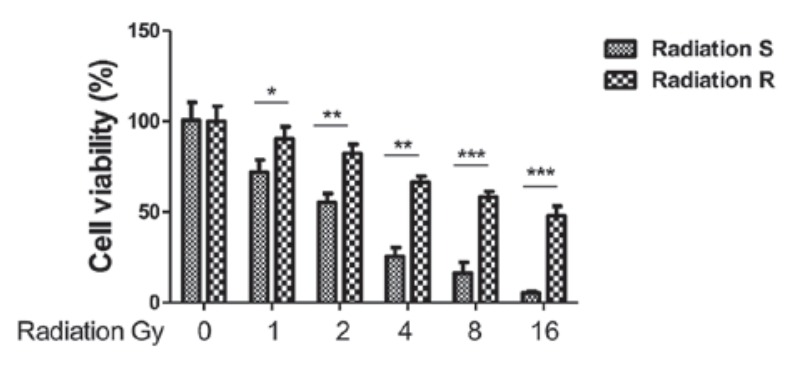

B

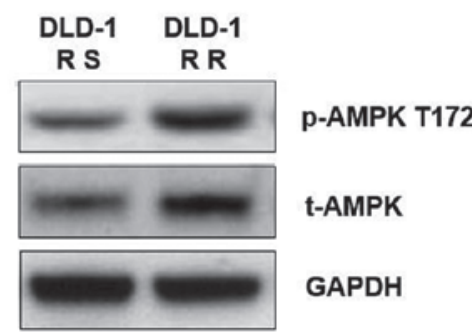

C

DLD-1 AMPK mRNA

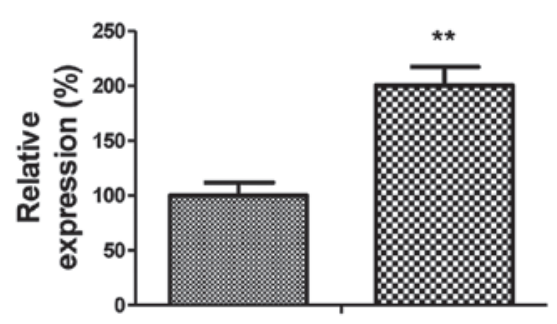

Radiation S \& Radiation $\mathbf{R}$

Figure 2. Radiation resistant DLD-1 cells exhibit elevated AMPK activity. (A) Generation of radiation resistant cells from DLD-1 cells. The pooled radioresistant clones were treated with radiation at indicated doses, followed by cell viability assay. (B) DLD-1 radiation sensitive and resistant cells were analyzed by western blotting to detect the protein levels of total AMPK and phospho-AMPK at the phosphorylation site on Threonine 172. (C) DLD-1 radiation sensitive and resistant cells were analyzed by reverse transcriptionquantitative polymerase chain reaction to detect the mRNA levels of total AMPK. Columns, mean of three independent experiments; bars, standard error. "P<0.05; ** $\mathrm{P}<0.01$; ${ }^{* * *} \mathrm{P}<0.001$. AMPK, adenosine monophosphate-activated kinase; t-AMPK, total AMPK; p-AMPK, phospho-AMPK; GAPDH, glyceraldehyde-3-phosphate dehydrogenase.

from 0-16 Gy for $24 \mathrm{~h}$. Cell viability assays demonstrated that DLD-1 radioresistant cells tolerated higher doses of radiation compared with radiosensitive cells. The $\mathrm{IC}_{50}$ of the resistant cells was $20 \mathrm{~Gy}$, which was $\sim 10$-fold greater than the $\mathrm{IC}_{50}$ of the radiosensitive cells, which exhibited significant inhibition of viability at 1-16 Gy (Fig. 2A). The phosphorylation of AMPK and total AMPK were upregulated in DLD-1 radioresistant cells (Fig. 2B) in addition to the mRNA level of AMPK (Fig. 2C; $\mathrm{P}<0.01$ ), consistent with the above results that AMPK pathway was upregulated in radiation resistant colon cancers.

Overexpression of AMPK in colon cancer cells contributes to radiation resistance. To address whether AMPK activation is involved in the radiosensitivity of colon cancer cells, the cell survival profiles of DLD-1 cells was analyzed in 


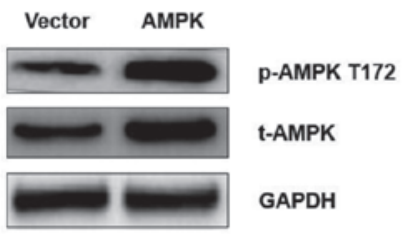

DLD-1

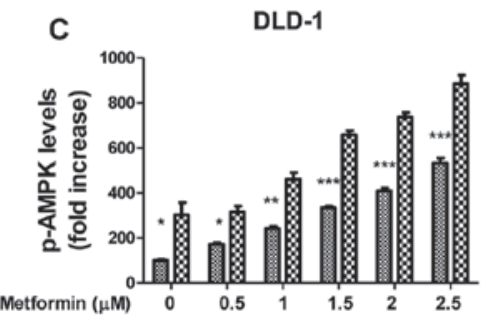

B
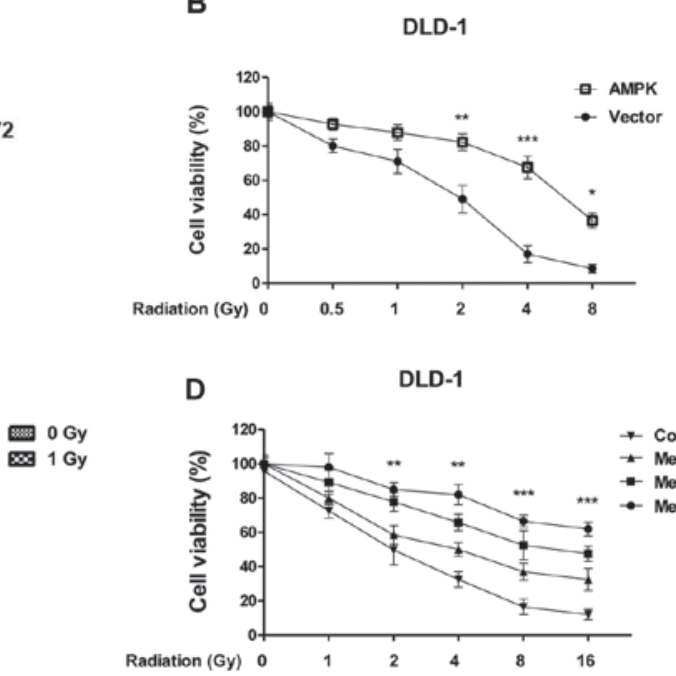

D

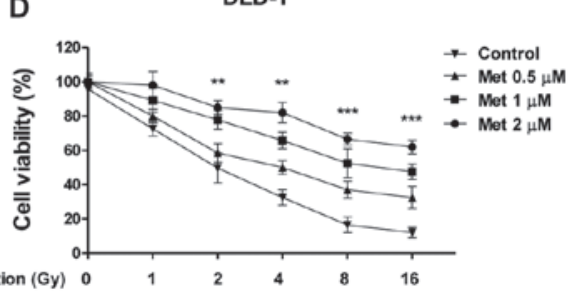

Figure 3. Overexpression of AMPK renders renal cancer cells resistance to radiation. (A) DLD-1 cells were transfected with AMPK or control vector for $48 \mathrm{~h}$, the expression of total AMPK and phospho-AMPK at T172 were measured by western blot analysis. GAPDH was a loading control. (B) DLD-1 cells were transfected with AMPK or control vector for $48 \mathrm{~h}$, followed by irradiation at $0,0.5,1,2,4$ and $8 \mathrm{~Gy}$. Then cells were analyzed by cell viability assay. (C) DLD-1 cells were treated with Metformin at the indicated concentrations for $1 \mathrm{~h}$, then the phospho-AMPK at T172 were measured by western blot, the relative intensities were showed as folds increase. (D) DLD-1 cells were treated with Metformin at the indicated concentrations for $1 \mathrm{~h}$, then cells were exposed to radiation at $0,1,2,4,8$ and $16 \mathrm{~Gy}$, followed by the measurements of cell viability. Columns, mean of three independent experiments; bars, standard error. "P $<0.05$ vs. control group; ${ }^{* *} \mathrm{P}<0.01$ vs. control group; ${ }^{* * *} \mathrm{P}<0.001$ vs. control group. AMPK, adenosine monophosphate-activated kinase; t-AMPK, total AMPK; p-AMPK, phospho-AMPK; GAPDH, glyceraldehyde-3-phosphate dehydrogenase; T172, phosphorylation site on Threonine 172.

A

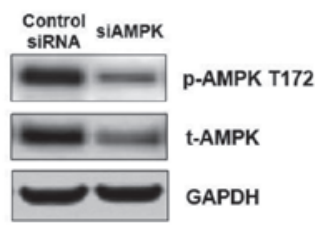

C

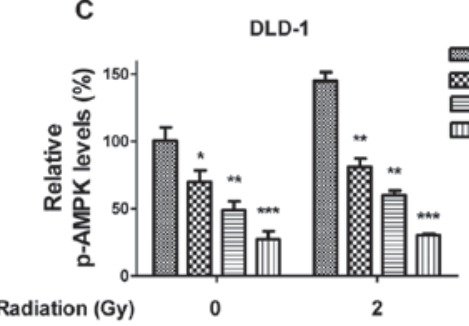

B

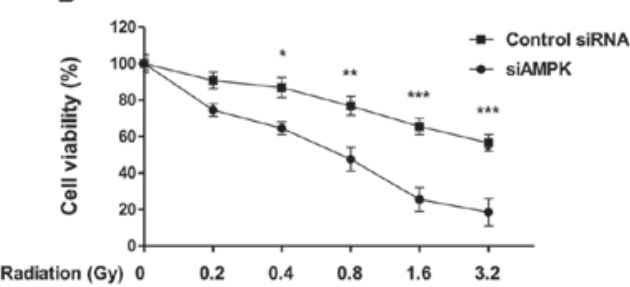

D

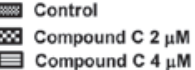
署 Compound $\mathrm{C} 2 \mu \mathrm{M}$ 孟 Compound $\mathrm{C} 8 \mu \mathrm{M}$

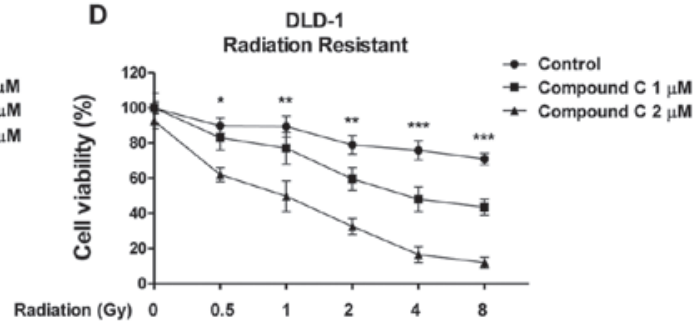

Figure 4. Inhibition of AMPK re-sensitizes radiation resistant cells. (A) DLD-1 radiation resistant cells were transfected siAMPK or control siRNA for $48 \mathrm{~h}$, the expression of t-AMPK and p-AMPK at T172 were measured by western blot analysis. GAPDH was a loading control. (B) Cells with siAMPK or control siRNA were treated with radiation at indicated doses, followed by cell viability assay. (C) Inhibition of the activity of AMPK by Compound C. DLD-1 radiation resistant cells were treated with Compound $\mathrm{C}$ at the indicated concentrations for $1 \mathrm{~h}$, then the p-AMPK at T172 were measured by western blot analysis, the relative intensities are presented as fold increase. (D) DLD-1 radiation resistant cells were treated with Compound C at the indicated concentrations for $1 \mathrm{~h}$, then cells were treated with radiation at indicated doses, followed by cell viability assay. Columns, mean of three independent experiments; bars, standard error. "P $<0.05$; ${ }^{* *} \mathrm{P}<0.01 ;{ }^{* * *} \mathrm{P}<0.001$. siRNA, small interfering RNA; AMPK, adenosine monophosphate-activated kinase; t-AMPK, total AMPK; p-AMPK, phospho-AMPK; GAPDH, glyceraldehyde-3-phosphate dehydrogenase.

cells that overexpressed AMPK. AMPK was successfully upregulated in cells transfected with the overexpression vector compared with the control (Fig. 3A); overexpression in colon cancer cells led to increased resistance to radiation treatments (Fig. 3B; $\mathrm{P}<0.01$ ), indicating activation of AMPK pathway contributes to radioresistance. To investigate further, DLD-1 cells were treated with metformin, which is a well-studied stimulator of AMPK pathway, at the indicated concentrations. Metformin alone $(0.5-2.5 \mu \mathrm{M})$ induced a dose-dependent activation of AMPK, it also significantly enhanced the activation of AMPK under radiation treatment (Fig. 4C; $\mathrm{P}<0.01$ with $2.5 \mu \mathrm{M}$ metformin). The effects of irradiation and metformin on DLD-1 cells survival. The surviving fraction of metformin pretreated-cells following radiation treatments 
were significantly higher compared with non-treated cells (Fig. 4D; P<0.01, following 10 Gy IR and $2 \mu \mathrm{M}$ metformin versus $0 \mu \mathrm{M}$ metformin), indicating that the activation of the AMPK pathway may have contributed to colon cancer cells resistance to radiation.

Inhibition of AMPK re-sensitizes radiation-resistant colon cancer cells. To investigate whether targeting the AMPK pathway could attenuate radiation resistance, molecular and biochemical inhibition of AMPK was achieved by knocking down expression of AMPK by siRNA and treatment with Compound C (CC) which is an inhibitor of AMPK pathway (Fig. 4A). In DLD-1 cells, knocking down of AMPK by anti-AMPK $\alpha$ subunit siRNA led to a significant suppression of cell survival rates following radiation (Fig. 4B), consistent with the above results that DLD-1 cells became resistant to radiation with the overexpression of AMPK (Fig. 3B). CC treatment inhibited the activities of AMPK (Fig. 4C) and the inhibition of AMPK with CC $(2,4$ and $8 \mu \mathrm{M})$ abolished the radiation-activated of AMPK, suggesting that blocking the AMPK pathway may be effective in resensitizing radioresistant cancer cells to radiation. To evaluate the effects of treating radioresistant colon cancer cells with the combination of AMPK inhibitor and radiation, DLD-1 radiation resistant cells were treated with CC ( 1 and $2 \mu \mathrm{M} \mathrm{CC})$. The cells were then treated with radiation at the indicated dosages. CC treatment in radiation resistant colon cancer cells significantly promoted the susceptibility to radiation at multiple doses and the AMPK pathway was significantly inhibited (Fig. 4D). Taken together, the results suggested that the combination of AMPK inhibitor and radiation showed a synergistic inhibitory effect on radioresistant colon cancer cells.

\section{Discussion}

A previous study reported that ionic radiation increased AMPK protein levels in lung cancer and breast cancer cells (12). In the present study, the importance of AMPK in radiation resistance in colon cancer cells was assessed and highlighted the clinical relevance by inhibition of AMPK, suggesting a therapeutic role of AMPK inhibitor for overcoming radiation resistance. Furthermore, RT-qPCR analysis demonstrated an induction of AMPK mRNA expression in radiation resistant cells, suggesting that the AMPK expression is regulated at the translational and transcriptional level. However, the detailed mechanisms for the downstream regulator of AMPK remain to be elucidated. A preliminary study suggested that IR stimulates AMPKb1/2 gene expression in HCT116 and H1299 cells in a p53-dependent manner (11): Putative p53 consensus binding sites were identified on the AMPKb1 and b2 promoters. The upstream regulators of AMPK in response to radiation therefore require further study.

Metformin is a widely used drug for treatment of type 2 diabetes and it activates AMPK leading to the induction of fatty acid oxidation and glucose uptake (13). In addition, it has been reported that chronic activation of AMPK may also induce the expression of muscle hexokinase and glucose transporters (Glut4) (14). The present study indicates that the activation of AMPK by metformin contributes to radiation resistance in colon cancer cells, suggesting that AMPK-mediated radioresistance may be affected by cancer metabolic regulators. Notably, inhibition of AMPK by CC re-sensitized radiation resistant cells.
AMPK has been reported to promote glycolysis of cancer cells as an adaptive response to metabolic stress (15). The present study provides evidence that the activated AMPK contributes to radioresistance in colon cancer cells. Since chemo- and radio-resistant cancer cells exhibit elevated levels of glycolysis, the present results indicates that AMPK-mediated radioresistance may be a result of up-regulation of glycolysis in cancer cells. Future studies should investigate the molecular mechanisms of this phenotype by measuring the glycolysis ratio of radioresistant cells and determining the signal pathway that regulates AMPK-mTOR-Akt in response to radiation. In conclusion, the present study indicated the important roles of AMPK pathway in radiation resistance in cancer cell lines and colon cancer patient samples and highlights a novel strategy for the development of therapeutic agents to reverse radiation resistance.

\section{Acknowledgements}

The authors would like to thank the staff and faculty members working in the Department of General Surgery, China Japan Union Hospital of Jilin University and Dr. Zhuo Liu for editorial assistance.

\section{References}

1. Rastogi RP, Richa, Kumar A, Tyagi MB and Sinha RP: Molecular mechanisms of ultraviolet radiation-induced DNA damage and repair. J Nucleic Acids 2010: 592980, 2010.

2. Pajonk F, Vlashi E and McBride WH. Radiation resistance of cancer stem cells: The 4 R's of radiobiology revisited. Stem Cells 28: 639-648, 2010.

3. Galanty Y, Belotserkovskaya R, Coates J, et al: Mammalian SUMO E3-ligases PIAS1 and PIAS4 promote responses to DNA double-strand breaks. Nature 462: 935-939, 2009.

4. Lavin MF: Ataxia-telangiectasia: From a rare disorder to a paradigm for cell signalling and cancer. Nat Rev Mol Cell Biol 9: 759-769, 2008.

5. Shen $\mathrm{C}$ and Houghton PJ: The mTOR pathway negatively controls ATM by up-regulating miRNAs. Proc Natl Acad Sci USA 110: 11869-11874, 2013

6. Hardie DG, Ross FA and Hawley SA: AMPK: A nutrient and energy sensor that maintains energy homeostasis. Nat Rev Mol Cell Biol 13: 251-262, 2012.

7. Gurumurthy S, Xie SZ, Alagesan B, et al: The Lkb1 metabolic sensor maintains haematopoietic stem cell survival. Nature 468: 659-663, 2010.

8. Hardie DG: AMPK: Positive and negative regulation, and its role in whole-body energy homeostasis. Curr Opin Cell Biol 33: 1-7, 2015.

9. Jones RG, Plas DR, Kubek S, et al: AMP-activated protein kinase induces a p53-dependent metabolic checkpoint. Mol Cell 18: 283-293, 2005.

10. Inoki K, Kim J and Guan KL: AMPK and mTOR in cellular energy homeostasis and drug targets. Annu Rev Pharmacol Toxicol 52: 381-400, 2012.

11. Sanli T, Storozhuk Y, Linher-Melville K, et al: Ionizing radiation regulates the expression of AMP-activated protein kinase (AMPK) in epithelial cancer cells: Modulation of cellular signals regulating cell cycle and survival. Radiother Oncol 102: 459-465, 2012.

12. Sanli T, Rashid A, Liu C, et al: Ionizing radiation activates AMP-activated kinase (AMPK): A target for radiosensitization of human cancer cells. Int J Radiat Oncol Biol Phys 78: 221-229, 2010.

13. Rena G, Pearson ER and Sakamoto K: Molecular mechanism of action of metformin: Old or new insights? Diabetologia 56: 1898-1906, 2013.

14. Habegger KM, Hoffman NJ, Ridenour CM, et al: AMPK enhances insulin-stimulated GLUT4 regulation via lowering membrane cholesterol. Endocrinology 153: 2130-2141, 2012.

15. Wu SB and Wei YH: AMPK-mediated increase of glycolysis as an adaptive response to oxidative stress in human cells: Implication of the cell survival in mitochondrial diseases. Biochim Biophys Acta 1822: 233-247, 2012. 